\title{
The cystine/glutamate antiporter: when too much of a good thing goes bad
}

\author{
Kathryn J. Reissner \\ Department of Psychology and Neuroscience Center, University of North Carolina, Chapel Hill, Chapel Hill, North Carolina, USA.
}

\begin{abstract}
Clutamate excitotoxicity represents a major cellular component of ischemic brain injury. In this issue of the $J \mathrm{Cl}$, Soria and colleagues reveal that the cystine/glutamate exchanger is an important source of excitotoxic glutamate in response to ischemia induced by oxygen and glucose deprivation. As the exchanger is a primary determinant of both extracellular glutamate and intracellular glutathione, the findings from this study not only provide important insight into the mechanisms of brain ischemia but also demonstrate the complexity of the yin and yang of glutamate homeostasis and cellular redox balance.
\end{abstract}

Ischemia-induced glutamate: tracing it to the source

Ischemia is defined as an insufficient blood supply and consequently results in oxygen deprivation and energy depletion in vascularized tissues. Ischemic brain injury most commonly occurs as the result of thrombolytic or embolic stroke but can occur in any condition in which blood or oxygen to the brain is restricted. Ischemic hypoxia in the brain rapidly leads to a series of deleterious cellular events, which culminate in injury and death to neurons, glia, and endothelial cells. The consequences of ischemic damage to neurons, in particular the resulting glutamatergic excitotoxicity, have been well described (for review, see refs. 1, 2). To date, very few effective interventions exist to mitigate brain ischemia-induced damage, which can be associated with a continuum of physiological consequences ranging from disability to death.

Conditions of oxygen deprivation in neural tissue lead to anoxic depolarization ( $\mathrm{AD}$, also referred to as peri-infarct depolarization) of membrane potentials, which results from overstimulation of glutamate receptors and a large glutamate-mediated inward current, referred to as either the AD current or ischemia- induced anoxic current $(3,4)$. However, the source and mechanism of production of excitotoxic glutamate, which mediates oxygen and glucose deprivation-induced (OGD-induced) neuronal damage, are not well defined.

In the current study, Soria et al. (5) used $\mathrm{AD}$ depolarization and the anoxic current in pyramidal neurons in acute cortical slices to investigate excitotoxic glutamate release in rodent models of ischemia. One very logical candidate for excitotoxic glutamate release is the induction of reverse transport by glutamate transporters. For example, high-affinity glutamate transporters typically function to move glutamate from the extracellular space into the cell; however, in the presence of decreased extracellular $\mathrm{Na}^{+}$(such as depolarization), these transporters can function in reverse, thereby releasing glutamate from the cell $(6,7)$. Interestingly, pharmacological inhibition of glutamate transporters had no effect on the OGD-induced anoxic current, indicating that reverse transport is an unlikely source of excess glutamate (Figure 1). In fact, transporter blockade magnified ischemiainduced damage, indicating that transporters were likely still working in the forward direction. Similarly, blockade of vesicular

\section{Related Article: p. 3645}

glutamate release with bafilomycin $\mathrm{A} 1$ had no effect on the anoxic current, although miniature excitatory postsynaptic currents (mEPSCs) were effectively blocked (Figure 1). Neither bafilomycin A1 nor tetanus toxin, which also blocks neurotransmitter exocytosis, affected OGD-induced neuronal damage. In contrast, inhibition of the cystine/glutamate exchanger (system $\mathrm{xc}^{-}$) with either S-4-carboxyphenylglycine (CPG) or sulfasalazine (SAS) dramatically reduced the amplitude of the anoxic current as well as OGD-associated neuronal damage (Figure 1). Soria and colleagues (5) went on to measure OGD-induced effects in mice lacking functional cystine/ glutamate exchange (Slc7a11sut mice) and found that these mice exhibited a reduction in anoxic currents and neuronal damage following ischemia, further supporting glutamate exchange as a source of excitotoxic glutamate.

\section{Exchanger-derived glutamate targets extrasynaptic NR2B- containing receptors}

These results from Soria and coworkers (5) intriguingly suggest that extracellular glutamate derived from the cystine/glutamate exchanger mediates neuronal damage induced by ischemia. Indeed, system $\mathrm{xc}^{-}$ has been well described as a major source of extrasynaptic glutamate (8). Release of glutamate by the exchanger is coupled with uptake of cystine at a 1:1 ratio. Cystine then serves as an intracellular precursor for the generation of the antioxidant glutathione. In this way, the exchanger also serves an important role in antioxidantmediated cellular protection (9-11).

While release of glutamate from the exchanger has been linked with stimulation of presynaptic inhibitory metabotropic glutamate receptors in the ventral striatum (12), evidence coupling this source of glutamate with stimulation of extrasynaptic excitatory receptors is lacking. Because it is known that NMDA currents mediate excitotoxic damage (13), 


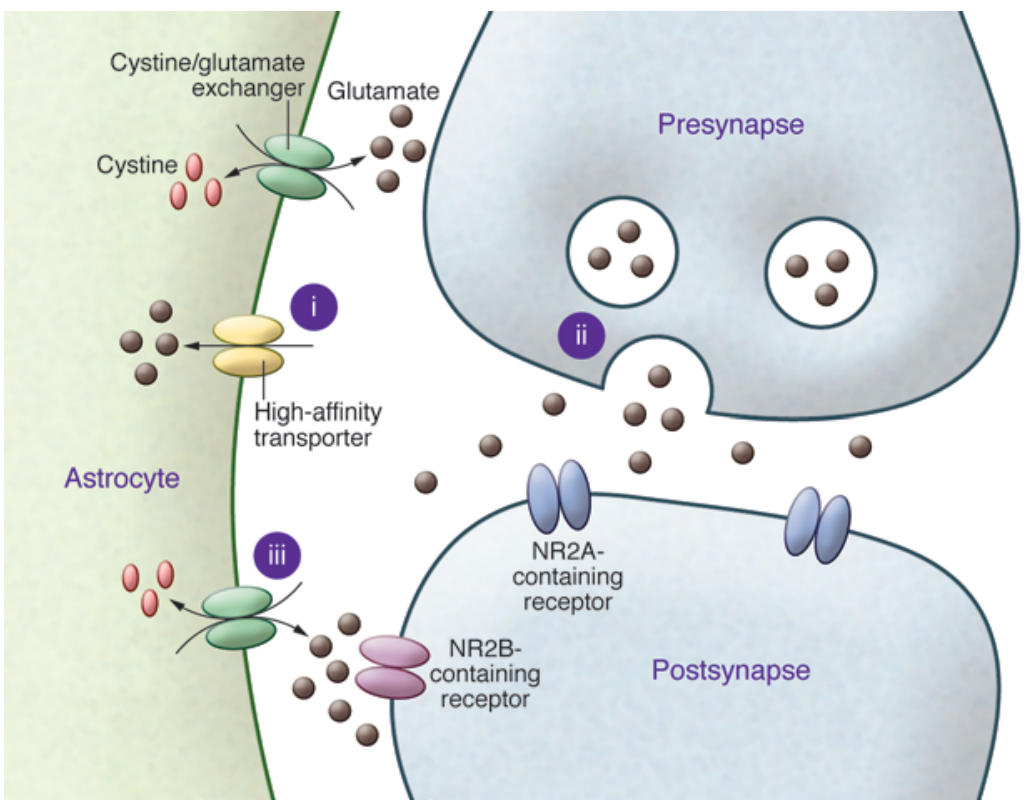

Soria et al. tested the hypothesis that exchanger-derived glutamate stimulates NMDA receptor-mediated (NMDARmediated) glutamate currents (5). Soria and colleagues applied cystine to acute slices and observed an inward current, which was blocked by administration of the NMDAR antagonist D-AP5. Moreover, this current was blocked by treatment with the system $\mathrm{xc}^{-}$antagonist CPG. Nonetheless, while the results of Soria and colleagues (5) indicate that NMDARs are stimulated by $\mathrm{xc}^{-}$-derived glutamate, they do not differentiate between synaptic and extrasynaptic NMDARs, which contribute differentially to postsynaptic signaling as well as to the adaptive and pathological consequences of NMDAR activation (14). To this point, pharmacological inhibition of extrasynaptic NR2B subunit-containing NMDARs impaired the anoxic current, whereas blockade of the synaptic NR2A subunit-containing receptors did not affect current amplitude, thus implicating extrasynaptic NR2B-containing receptors as the likely mediators of glutamate excitotoxicity. NR2B antagonists ifenprodil and RO256981 reduced the anoxic current, while NR2A antagonists did not alter the current without effect. Moreover, NR2A antagonists had no effect on OGD-mediated neuronal death, while inhibitors of the extrasynaptic NR2B-containing receptors were protective against cell death subsequent to OGD (5).

\section{System $\mathrm{xc}^{-}$is upregulated following ischemia}

Collectively, the results from Soria and colleagues indicate that anoxic currents and neuronal damage induced by OGD are mediated by glutamate derived from system $\mathrm{xc}^{-}$via activation of NR2B-containing extrasynaptic NMDARs (5). But, is the exchanger stimulated by ischemia? Real-time quantitative PCR and Western blot analysis of the catalytic subunit of the exchanger $(\mathrm{xCT})$ indicated that mRNA and protein were markedly upregulated 1 hour and 4 hours, respectively, following chemical ischemia in culture. Ischemia also induced enhanced functional transport of $\left[{ }^{14} \mathrm{C}\right]$ L-cystine. Additionally, Soria and colleagues induced transient focal ischemia in a rat model of middle cerebral artery occlusion (MCAO) and measured $\mathrm{xCT}$ function by radioligand imaging via PET (5). Acute ipsilateral MCAO resulted in tracer accumulation, supporting ischemia-induced upregulation of the exchanger in vivo.

\section{Perspectives}

In sum, the study by Soria et al. (5) identifies the cystine/glutamate exchanger as a major source of excitotoxic glutamate during ischemia in culture. In response to oxidative stress, both expression and activity of the cysteine/glutamate exchanger is upregulated (15); hence, this process may represent an adaptive attempt to compensate for ischemia-induced oxidative stress. Ironically, this potentially
Figure 1. Systematic analysis of the source of excitotoxic glutamate during ischemic brain injury. Excitotoxic glutamate (brown circles) released during oxygen and glucose deprivation in culture could potentially derive from reverse transport from high-affinity glutamate transporters ( $\mathrm{i}$, yellow), vesicular glutamate release (ii), or the cystine/glutamate exchanger (iii, green). Exchange of glutamate release for cystine uptake by the exchanger is indicated with bidirectional arrows. Soria and colleagues have demonstrated that glutamate released from the cystine/glutamate exchanger activates extrasynaptic NR2B-containing receptors (iii, purple), as opposed to synaptic NR2A-containing receptors (blue).

protective mechanism leads to damaging consequences. For example, the cystine pro-drug $\mathrm{N}$-acetylcysteine (NAC) activates system $\mathrm{xc}^{-}$and has previously been reported to have protective effects against brain ischemia $(16,17)$; therefore, NAC has been proposed as a possible target for the treatment of ischemic brain injury (18). However, the study by Soria and colleagues (5) clearly indicates that caution must be taken when considering the use of an activator of system $\mathrm{xc}^{-}$to reduce ischemia-associated damage.

Finally, it is important to note that pharmacological inhibition or genetic deletion of system $\mathrm{xc}^{-}$reduced, but did not completely block, the anoxic current and ischemiainduced cell death promoted by OGD, raising the possibility that glutamate from additional sources also contributes. Indeed, there are a number of additional nonvesicular sources of glutamate (for review see ref. 10); therefore, continued study into the mechanisms of glutamate release in response to ischemia is warranted for the development of more effective treatment strategies.

This study, together with other recent reports, challenges the simple view that in cases of pathophysiology, system $\mathrm{xc}^{-}$predominantly functions in an antioxidative, protective role. Quite the opposite: this complex system provides an intriguing example of how, despite the critical benefits of glutamate release in exchange for uptake of cystine, too much of a good thing really is a bad thing. 


\section{Acknowledgments}

This work was supported by NIH grant DA031790 (to K.J. Reissner).

Address correspondence to: Kathryn J. Reissner, University of North Carolina, Chapel Hill, CB 3270, 235 E. Cameron Ave., Chapel Hill, North Carolina 27599, USA. Phone: 919.843.9112; E-mail: reissner@unc.edu.

1. Tuttolomondo A, et al. Neuron protection as a therapeutic target in acute ischemic stroke. Curr Top Med Chem. 2009;9(14):1317-1334.

2. Hertz L. Bioenergetics of cerebral ischemia: a cellular perspective. Neuropharmacology. 2008;55(3):289-309.

3. Hamann M, Rossi DJ, Mohr C, Andrade AL, Attwell D. The electrical response of cerebellar Purkinje neurons to simulated ischaemia. Brain. 2005;128(pt 10):2408-2420.

4. Weilinger NL, Tang PL, Thompson RJ. Anoxiainduced NMDA receptor activation opens pannexin channels via Src family kinases. J Neurosci. 2012;32(36):12579-12588.

5. Soria FN, et al. Extrasynaptic glutamate release through cystine/glutamate antiporter contributes to ischemic damage. J Clin Invest.
2014;124(8):3645-3655.

6. Grewer C, Gameiro A, Zhang Z, Tao Z, Braams S, Rauen T. Glutamate forward and reverse transport: from molecular mechanism to transportermediated release after ischemia. IUBMB Life. 2008;60(9):609-619.

7. Camacho A, Massieu L. Role of glutamate transporters in the clearance and release of glutamate during ischemia and its relation to neuronal death. Arch Med Res. 2006;37(1):11-18.

8. Baker DA, Xi ZX, Shen H, Swanson CJ, Kalivas PW. The origin and neuronal function of in vivo nonsynaptic glutamate. J Neurosci. 2002;22(20):9134-9141.

9. Shih AY, Erb H, Sun X, Toda S, Kalivas PW, Murphy TH. Cystine/glutamate exchange modulates glutathione supply for neuroprotection from oxidative stress and cell proliferation. J Neurosci. 2006;26(41):10514-10523.

10. Bridges R, Lutgen V, Lobner D, Baker DA. Thinking outside the cleft to understand synaptic activity: contribution of the cystine-glutamate antiporter (System xc') to normal and pathological glutamatergic signaling. Pharmacol Rev. 2012;64(3):780-802.

11. Lewerenz J, et al. The cystine/glutamate antiporter system $\mathrm{x}(\mathrm{c})(-)$ in health and disease: from molecular mechanisms to novel therapeutic opportunities. Antioxid Redox Signal. 2013;18(5):522-555.
12. Moran MM, McFarland K, Melendez RI, Kalivas PW, Seamans JK. Cystine/glutamate exchange regulates metabotropic glutamate receptor presynaptic inhibition of excitatory transmission and vulnerability to cocaine seeking. J Neurosci. 2005;25(27):6389-6393.

13. Parsons MP, Raymond LA. Extrasynaptic NMDA receptor involvement in central nervous system disorders. Neuron. 2014;82(2):279-293.

14. Hardingham GE, Bading H. Synaptic versus extrasynaptic NMDA receptor signalling: implications for neurodegenerative disorders. Nat Rev Neurosci. 2010;11(10):682-696.

15. Conrad M, Sato H. The oxidative stress-inducible cystine/glutamate antiporter, system x (c) (-): cystine supplier and beyond. Amino Acids. 2012;42(1):231-246.

16. Knuckey NW, Palm D, Primiano M, Epstein $\mathrm{MH}$, Johanson CE. N-acetylcysteine enhances hippocampal neuronal survival after transient forebrain ischemia in rats. Stroke. 1995 ; 26(2):305-310.

17. Cuzzocrea S, et al. Beneficial effects of n-acetylcysteine on ischaemic brain injury. Br J Pharmacol.2000;130(6):1219-1226.

18. Bavarsad Shahripour R, Harrigan MR, Alexandrov AV. N-acetylcysteine (NAC) in neurological disorders: mechanisms of action and therapeutic opportunities. Brain Behav. 2014;4(2):108-122. 